H. ZHONG

KODAI MATH. J.

18 (1995), 250-259

\title{
ENTIRE FUNCTIONS THAT SHARE ONE VALUE WITH THEIR DERIVATIVES
}

\author{
HUALIANG ZHONG
}

\begin{abstract}
The paper generalizes a result of [2] and makes an example which shows that the generalization is precise. Also we get similar conclusions in other cases.
\end{abstract}

\section{§1. Introduction}

We say that nonconstant meromorphic functions $f$ and $g$ share the value $a$ provided that $f(z)=a$ if and only if $g(z)=a$. We will state whether the shared value is by $\mathrm{CM}$ (counting mulitiplicities) or by IM (ignoring multiplicities).

L. Rubel and C.C. Yang proved the following theorem:

THEOREM $\mathrm{A}^{[1]}$. Let $f(z)$ be a nonconstant entire function. If $f$ and $f^{\prime}$ share two distinctive valves $a$ and $b I M$, then $f \equiv f^{\prime}$.

1986, Jank, Mues and Volkman proved:

THEOREM $\mathrm{B}^{[2]}$. Let $f(z)$ be a nonconstant entire function. If $f$ and $f^{\prime}$ share the value $a(a \neq 0)$, and $f^{\prime \prime}(z)=a$ when $f(z)=a$, then $f \equiv f^{\prime}$.

It is asked naturally whether the $f^{\prime \prime}$ of Theorem B can be simply replaced by $f^{(k)}(k \geqq 3)$. We make an example which shows that the answer of this question is negative.

Let $k$ be a positive integer $(k \geqq 3)$ and let $\omega(\neq 1)$ be a $(k-1)$-th root of unity. Set $g(z)=e^{\omega z}+\omega-1$. It is easy to know that $g, g^{\prime}$ and $g^{(k)}$ share the value $\omega$ $\mathrm{CM}$, but $g \not \equiv g^{\prime}$ and $g \not \equiv g^{(k)}$.

Between the example and Theorem $B$ we will prove the following results.

THEOREM 1. Let $f(z)$ be a nonconstant entire function. If $f$ and $f^{\prime}$ share the value $a(a \neq 0) C M$, and $f^{(n)}(z)=f^{(n+1)}(z)=a(n \geqq 1)$ when $f(z)=a$, then $f \equiv f^{(n)}$.

Received Mach 7, 1994; revised July 26, 1994. 
It is obvious that Theorem B is a special case of Theorem 1 .

Before stating Theorem 2, we need a slight general notation about the "share". We assume that the reader is familiar with the usual notations and fundamental results of Nevanlinna's theory of meromorphic functions (see, e.g. [3], [8]). In particular, $S(r, f)$ will denote any quantity that satisfies $S(r, f)$ $=o(1) T(r, f)$ as $r \rightarrow+\infty$, possibly outside a set $e$ of $r$ of finite linear measure.

Let $E=E\left\{f_{1}, f_{2}, \cdots, f_{n} ;\left(a_{1}, a_{2}, \cdots, a_{n}\right)\right\}=\left(\bigcup_{j=1}^{n} f_{j}^{-1}\left(a_{\jmath}\right)\right) \backslash\left(\cap_{\jmath=1}^{n} f_{j}^{-1}\left(a_{j}\right)\right)$ and let $N_{E}\left(r, 1 /\left(f_{i}-a_{\imath}\right)\right)$ denote the counting function of those $a_{i}$-points of $f_{\imath}$ which belong to the set $E, i=1,2, \cdots, n$. We introduce the following definitions:

DEFINITION 1. Two nonconstant meromorphic functions $f$ and $g$ share the value $a \mathrm{CMN}$ (or IMN) if $f$ and $g$ share the value $a \mathrm{CM}$ (or IM) outside the set $E$ where $E(=E\{f, g ;(a, a)\})$ satisfies that $N_{E}(r, 1 /(f-a))=S(r, f)$ and $N_{E}(r,(1 / g-a))=S(r, g)$.

THEOREM 2. Let $f(z)$ be a nonconstant entire function, $f, f^{(n)}(n \geqq 1)$ share the value $a(\neq 0)$ IMN, and $f^{\prime}(z)=f^{(n+1)}(z)=a$ when $f(z)=a$. If for any set $e$ of finite linear measure

$$
\lim _{\substack{r \rightarrow \infty \\ r \notin e}} \frac{N_{E}\left(r, \frac{1}{f^{(n+1)}-a}\right)}{N\left(r, \frac{1}{f^{(n+1)}-a}\right)} \neq \frac{1}{2}
$$

where $E=E\left\{f, f^{(n+1)} ;(a, a)\right\}$, then $f \equiv f^{(n)}$.

We don't know whether it is possible to get rid of the condition (1.1) in Theorem 2.

DEFINITION 2. Meromorphic functions $f_{1}, f_{2}, \cdots, f_{n}$ share the array $\left(a_{1}, a_{2}\right.$, $\left.\cdots, a_{n}\right) \mathrm{CM}$ provided that $f_{1}^{-1}\left(a_{1}\right)=f_{j}^{-1}\left(a_{j}\right)(1 \leqq j \leqq n)$ by counting mulitiplicities. Similarly we say $f_{1}, f_{2}, \cdots, f_{n}$ share the array $\left(a_{1}, a_{2}, \cdots, a_{n}\right)$ CMN (or IMN) if $f_{1}, f_{2}, \cdots, f_{n}$ share the array $\left(a_{1}, a_{2}, \cdots, a_{n}\right)$ CM (or IM) outside the set $E=E\left\{f_{1}, f_{2}, \cdots, f_{n},\left(a_{1}, a_{2}, \cdots, a_{n}\right)\right\}$ where $E$ satisfies that $N_{E}\left(r, 1 /\left(f_{j}-a_{j}\right)=\right.$ $S\left(r, f_{j}\right), j=1,2, \cdots, n$.

There is an example which shows that the condition of Theorem B can't be replaced by the condition that $f, f^{\prime}$ and $f^{\prime \prime}$ share the array $(a, a, b) \mathrm{CM}$. Let $g(z)=e^{2 z}+1$. It is easy to know that $g, g^{\prime}, g^{\prime \prime}$ share $(2,2,4)$ and $g \not \equiv g^{\prime}$. However we can make the following conclusion.

THEOREM 3. Let $f(z)$ be a nonconstant entire function. If $f, f^{\prime}, f^{\prime \prime}$ share the array $(a, b, b) I M N$, where $a b \neq 0$, then $f-a \equiv f^{\prime}-b$.

\section{§ 2. Lemmas}

LEMMA 1. Let $f(z)$ be a nonconstant meromorphic function and let $b_{\jmath}(\jmath=1$, $2, \cdots, q)$ be $q$ distinctive finite numbers. Then for any integer $k \geqq 0$, 


$$
\sum_{j=1}^{q} m\left(r, \frac{1}{f^{(k)}-b_{\jmath}}\right) \leqq m\left(r, \frac{1}{f^{(k+1)}}\right)+S(r, f)
$$

where $S(r, f)=o(1) T(r, f)$ as $r \rightarrow \infty$, possibly outside a set $e$ of $r$ of finte linear measure.

This lemma can be simply derived from p. 16 of [3].

LEMMA 2. Suppose that entire functions $f, f^{\prime}$ share the value a $(\neq 0) C M N$. Then

$$
T(r, f) \leqq 2 N\left(r, \frac{1}{f-a}\right)+S(r, f) .
$$

Proof. Since, from Lemma 1, we know that

$$
\begin{gathered}
m\left(r, \frac{1}{f-a}\right)+m\left(r, \frac{1}{f^{\prime}-a}\right) \leqq m\left(r, \frac{1}{f^{\prime}}\right)+m\left(r, \frac{1}{f^{\prime}-a}\right)+S(r, f) \\
\leqq m\left(r, \frac{1}{f^{\prime \prime}}\right)+S(r, f) \leqq T\left(r, f^{\prime \prime}\right)+S(r, f),
\end{gathered}
$$

it follows that

$$
T(r, f)+T\left(r, f^{\prime}\right) \leqq 2 N\left(r, \frac{1}{f-a}\right)+T\left(r, f^{\prime \prime}\right)+S(r, f) .
$$

By using $T\left(r, f^{\prime \prime}\right) \leqq T\left(r, f^{\prime}\right)+S(r, f)$, we get

$$
T(r, f) \leqq 2 N\left(r, \frac{1}{f-a}\right)+S(r, f) .
$$

LEMMA 3. Suppose that nonconstant entire functions $f, f^{(k)}(k \geqq 1)$ share the array $(a, b)$ IMN, $f^{\prime}(z)=f^{(k+1)}(z)=b$ when $f(z)=a$, where $a b \neq 0$. If $f^{(k)}-b \neq$ $f-a$, then the following statements hold:

$$
\begin{gathered}
2 N\left(r, \frac{1}{f-a}\right) \leqq T(r, f)+S(r, f) \\
T(r, f) \leqq 2 N\left(r, \frac{1}{f-a}\right)+S(r, f) . \\
N\left(r, \frac{1}{f^{(k+1)}}\right) \leqq S(r, f) .
\end{gathered}
$$

Proof. From the conditions of the lemma, we see that if $z_{0} \in f^{-1}(a) \cap$ $\left(f^{(k)}\right)^{-1}(b)$, then $z_{0}$ must be a common zero-point of $f-a, f^{(k)}-b$ and $\left(f^{(k)}-b\right)$ $-(f-a)$ with multiplicities 1,1 and $\gamma(\geqq 2)$ respectively. Then 


$$
\begin{aligned}
2 N\left(r, \frac{1}{f-a}\right) & \leqq N\left(r, \frac{1}{\left(f^{(k)}-b\right)-(f-a)}\right)+S(r, f) \\
& \leqq T\left(r, f^{(k)}-f\right)+S(r, f) \leqq T(r, f)+S(r, f) .
\end{aligned}
$$

Since, by using Lemma 1 , we get that

$$
\begin{aligned}
m\left(r, \frac{1}{f-a}\right)+m\left(r, \frac{1}{f^{(k)}-b}\right) & \leqq m\left(r, \frac{1}{f^{(k)}}\right)+m\left(r, \frac{f^{(k)}}{f-a}\right)+\log 2+m\left(r, \frac{1}{f^{(k)}-b}\right) \\
& \leqq m\left(r, \frac{1}{f^{(k+1)}}\right)+S(r, f),
\end{aligned}
$$

it is deduced that

$$
\begin{aligned}
T(r, f)+T\left(r, f^{(k)}\right) & \leqq N\left(r, \frac{1}{f-a}\right)+N\left(r, \frac{1}{f^{(k)}-b}\right)+m\left(r, \frac{1}{f^{(k+1)}}\right)+S(r, f) \\
& \leqq 2 N\left(r, \frac{1}{f-a}\right)+T\left(r, f^{(k+1)}\right)-N\left(r, \frac{1}{f^{(k+1)}}\right)+S(r, f)
\end{aligned}
$$

So

$$
T(r, f) \leqq 2 N\left(r, \frac{1}{f-a}\right)+S(r, f) .
$$

By using (2.4) and (2.6), we get (2.3).

Lemma $4^{[3]}$. Let $f(z)$ be an entire function. Then

$$
T(r, f) \leqq \bar{N}\left(r, \frac{1}{f}\right)+\bar{N}\left(r, \frac{1}{f-a}\right)+S(r, f) \quad \text { where } a \neq 0 .
$$

LEMMA 5. If entire functions $f, f^{\prime}$ and $f^{\prime \prime}$ share the array $(a, b, b) I M N$ $(a b \neq 0), z_{0} \in f^{-1}(a) \cap\left(f^{\prime}\right)^{-1}(b) \cap\left(f^{\prime \prime}\right)^{-1}(b)$, set

$$
H(z)=\frac{f^{\prime}}{f-a}-\frac{f^{\prime \prime}}{f^{\prime}-b} \quad K(z)=\frac{f^{\prime \prime}-f^{\prime}}{f-a},
$$

then

$$
\begin{gathered}
H(z)=\frac{1}{2}\left(1-\frac{f^{\prime \prime}\left(z_{0}\right)}{b}\right)+O\left\{\left(z-z_{0}\right)\right\} \\
K(z)=\frac{f^{\prime \prime}\left(z_{0}\right)}{b}-1+O\left\{\left(z-z_{0}\right)\right\} .
\end{gathered}
$$

Proof. Since the Taylor expansion of $f(z)$ about $z_{0}$ is

$$
f(z)=a+b\left(z-z_{0}\right)+\frac{b}{2 !}\left(z-z_{0}\right)^{2}+\frac{f^{\prime \prime \prime}\left(z_{0}\right)}{3 !}\left(z-z_{0}\right)^{3}+\cdots,
$$

by an elementary calculation, we can obtain (2.7) and (2.8). 


\section{§ 3. Proof of Theorem 1}

From Theorem B, we know that Theorem 1 is valid for $n=1$. Next we suppose that $n \geqq 2$. Set

$$
\varphi(z)=\frac{f^{(n+1)}(z)-f^{(n)}(z)}{f(z)-a}, \quad \phi(z)=\frac{f^{(n+1)}(z)-f^{(n)}(z)}{f^{\prime}(z)-a} .
$$

Then

$$
m(r, \varphi)=S(r, f), \quad m(r, \phi)=S(r, f) .
$$

Suppose $z_{1}$ is an $a$-point of $f(z)$. Then the Taylor expansion of $f(z)$ about $z_{1}$ is

$$
\begin{aligned}
f(z)= & a+a\left(z-z_{1}\right)+\frac{f^{\prime \prime}\left(z_{1}\right)}{2 !}\left(z-z_{1}\right)^{2}+\cdots+\frac{f^{(n-1)}\left(z_{1}\right)}{(n-1) !}\left(z-z_{1}\right)^{n-1} \\
& +\frac{a}{n !}\left(z-z_{1}\right)^{n}+\frac{a}{(n+1) !}\left(z-z_{1}\right)^{n+1}+\cdots
\end{aligned}
$$

Hence

$$
\begin{gathered}
\frac{f^{(n)}}{f-a}=\frac{1}{z-z_{1}}+\left(1-\frac{f^{\prime \prime}\left(z_{1}\right)}{2 a}\right)+O\left\{\left(z-z_{1}\right)\right\} \\
\frac{f^{(n+1)}}{f-a}=\frac{1}{z-z_{1}}+\frac{1}{a}\left[f^{(n+2)}\left(z_{1}\right)-\frac{f^{\prime \prime}\left(z_{1}\right)}{2}\right]+O\left\{\left(z-z_{1}\right)\right\} .
\end{gathered}
$$

It follows from (3.3) and (3.4) that

$$
\varphi(z)=\frac{f^{(n+2)}\left(z_{1}\right)-a}{a}+O\left\{\left(z-z_{1}\right)\right\} .
$$

Since the pole points of $\varphi(z)$ come from the $a$-points of $f(z)$, we see from (3.5) that

$$
N(r, \varphi)=0 .
$$

Also we know from (3.2) that

$$
\frac{f^{(n)}}{f^{\prime}-a}=\frac{a}{f^{\prime \prime}\left(z_{1}\right)} \cdot \frac{1}{\left(z-z_{1}\right)}+\frac{1}{f^{\prime \prime}\left(z_{1}\right)}\left[f^{(n+1)}\left(z_{1}\right)-\frac{a f^{\prime \prime \prime}\left(z_{1}\right)}{2 f^{\prime \prime}\left(z_{1}\right)}\right]+O\left\{\left(z-z_{1}\right)\right\}
$$

and that

$$
\frac{f^{(n+1)}}{f^{\prime}-a}=\frac{a}{f^{\prime \prime}\left(z_{1}\right)} \cdot \frac{1}{\left(z-z_{1}\right)}+\frac{1}{f^{\prime \prime}\left(z_{1}\right)}\left[f^{(n+2)}\left(z_{1}\right)-\frac{a f^{\prime \prime \prime}\left(z_{1}\right)}{2 f^{\prime \prime}\left(z_{1}\right)}\right]+O\left\{\left(z-z_{1}\right)\right\} .
$$

Therefore

$$
\phi(z)=\frac{f^{(n+2)}\left(z_{1}\right)-a}{f^{\prime \prime}\left(z_{1}\right)}+O\left\{\left(z-z_{1}\right)\right\}
$$

Thus 


$$
N(r, \phi)=0 .
$$

Combining (3.6), (3.8) with (3.1), we see that

$$
T(r, \varphi)=S(r, f), T(r, \phi)=S(r, f) .
$$

From (3.5) and (3.7), it follows that

$$
\frac{\varphi(z)}{\psi(z)}=\frac{f^{\prime \prime}\left(z_{1}\right)}{a}+O\left\{\left(z-z_{1}\right)\right\}
$$

Set $H(z)=\frac{f^{(n)}(z)-f^{\prime}(z)}{f(z)-a}$. It is easy to know that

$$
T(r, H) \leqq S(r, f) .
$$

From (3.2), we deduce that

$$
H(z)=\left[1-\frac{f^{\prime \prime}\left(z_{1}\right)}{a}\right]+O\left\{\left(z-z_{1}\right)\right\} .
$$

Hence

$$
H(z)+\frac{\varphi(z)}{\phi(z)}=1+O\left\{\left(z-z_{1}\right)\right\} .
$$

Next we consider the following two cases:

(i) Suppose that $H(z)+\varphi(z) / \psi(z) \not \equiv 1$. Set $L(z)=H(z)+\varphi(z) / \psi(z)-1$. Combining (3.9), (3.11) and (3.13), we deduce that

$$
N\left(r, \frac{1}{f-a}\right) \leqq N\left(r, \frac{1}{L}\right) \leqq T(r, L) \leqq S(r, f) .
$$

By using Lemma 2 , we get $T(r, f) \leqq S(r, f)$. It follows that $f(z)$ is a polynomial. So $f$ and $f^{\prime}$ can not share the value $a \mathrm{CM}$ which contradicts the condition of Theorem 1.

(ii) Let $H(z)+(\varphi(z) / \psi(z)) \equiv 1$. Then

Thus $f \equiv f^{(n)}$.

$$
\frac{f^{(n)}(z)-f^{\prime}(z)}{f(z)-a}+\frac{f^{\prime}(z)-a}{f(z)-a} \equiv 1
$$

\section{§4. Proof of Theorem 2}

Assume that $f \not \equiv f^{(n)}$. From Lemma 3, we see that

$$
T(r, f) \leqq 2 m\left(r, \frac{1}{f-a}\right)+S(r, f) .
$$

Hence

$$
T\left(r, f^{(n)}\right) \leqq T(r, f)+S(r, f) \leqq 2 m\left(r, \frac{1}{f-a}\right)+S(r, f) \leqq 2 m\left(r, \frac{1}{f^{(n)}}\right)+S(r, f) .
$$


Thus

$$
2 N\left(r, \frac{1}{f^{(n)}}\right) \leqq T\left(r, f^{(n)}\right)+S(r, f) .
$$

Now suppose that $f^{(n)} \equiv f^{(n+1)}$. Then $f^{(n)}(z)=c e^{z}$ and $f^{\prime}(z)-f(z)=c_{0} z^{n-1}+$ $\cdots+c_{n-2} z+c_{n-1}$ where $c$ and $c_{\imath}(i=0,1, \cdots, n-1)$ are constants. If $c \neq 0$, then $f^{(n)}$ has infinitely many $a$-points and there exist infinitely many points $z$ such that $f(z)=f^{\prime}(z)$. It is deduced that $c_{i}=0(i=0, \cdots, n-1)$ and that $f \equiv f^{\prime}$, which contradicts the assumption of $f \not \equiv f^{(n)}$. If $c=0$, then $f$ is a polynomial whose degree is at most $n-1$. Since $f$ is nonconstant, it has necessarily an $a$-point. By the assumption, it is also an $a$-point of $f^{(n+1)}(\equiv 0)$. This is a contradiction. So we assume that $f^{(n)} \neq f^{(n+1)}$, then

$$
\begin{aligned}
N\left(r, \frac{1}{f-a}\right) & \leqq N\left(r, \frac{1}{\frac{f^{(n+1)}}{f^{(n)}}-1}\right)+S(r, f) \leqq T\left(r, \frac{f^{(n+1)}}{f^{(n)}}\right)+S(r, f) \\
& \leqq N\left(r, \frac{1}{f^{(n)}}\right)+S(r, f) .
\end{aligned}
$$

Combining (4.3), (4.4) and (2.2), we get

$$
T(r, f) \leqq T\left(r, f^{(n)}\right)+S(r, f) .
$$

On the other hand, it follows from (2.1) that

So

$$
\begin{aligned}
2 N\left(r, \frac{1}{f^{(n)}-a}\right) & =2 N\left(r, \frac{1}{f-a}\right)+S(r, f) \leqq T(r, f)+S(r, f) \\
& \leqq T\left(r, f^{(n)}\right)+S(r, f) .
\end{aligned}
$$

$$
T\left(r, f^{(n)}\right) \leqq 2 m\left(r, \frac{1}{f^{(n)}-a}\right)+S(r, f) .
$$

Combining with (4.2), we deduce from Lemma 1 that

$$
m\left(r, \frac{1}{f^{(n+1)}}\right) \geqq m\left(r, \frac{1}{f^{(n)}}\right)+m\left(r, \frac{1}{f^{(n)}-a}\right)-S(r, f) \geqq T\left(r, f^{(n)}\right)-S(r, f) .
$$

Hence

$$
T\left(r, f^{(n)}\right) \leqq T\left(r, f^{(n+1)}\right)+S(r, f) .
$$

By using Lemma 3 and Lemma 4, we see that

$$
\begin{aligned}
T\left(r, f^{(n+1)}\right) & \leqq \bar{N}\left(r, \frac{1}{f^{(n+1)}}\right)+\bar{N}\left(r, \frac{1}{f^{(n+1)}-a}\right)+S(r, f) \\
& \leqq \bar{N}\left(r, \frac{1}{f^{(n+1)}-a}\right)+S(r, f) .
\end{aligned}
$$

Hence 


$$
N\left(r, \frac{1}{f^{(n+1)}-a}\right)=\bar{N}\left(r, \frac{1}{f^{(n+1)}-a}\right)+S(r, f) .
$$

From (4.10), (4.9), (4.5) and (2.1) we get

$$
2 N\left(r, \frac{1}{f-a}\right) \leqq \bar{N}\left(r, \frac{1}{f^{(n+1)}-a}\right)+S(r, f) .
$$

Also we know that

$$
\bar{N}\left(r, \frac{1}{f^{(n+1)}-a}\right) \leqq T\left(r, f^{(n+1)}\right) \leqq T(r, f)+S(r, f) \leqq 2 N\left(r, \frac{1}{f-a}\right)+S(r, f)
$$

and

$$
\begin{aligned}
2 N\left(r, \frac{1}{f-a}\right) & =2 \bar{N}\left(r, \frac{1}{f-a}\right)+S(r, f) \\
& =2\left[\bar{N}\left(r, \frac{1}{f^{(n+1)}-a}\right)-\bar{N}_{E}\left(r, \frac{1}{f^{(n+1)}-a}\right)\right]+S(r, f),
\end{aligned}
$$

where $E=E\left\{f, f^{(n+1)} ;(a, a)\right\}$.

We deduce from (4.12) and (4.14) that

$$
\bar{N}\left(r, \frac{1}{f^{(n+1)}-a}\right) \leqq 2 \bar{N}_{E}\left(r, \frac{1}{f^{(n+1)}-a}\right)+S(r, f),
$$

and from (4.13), (4.14) that

$$
\bar{N}\left(r, \frac{1}{f^{(n+1)}-a}\right) \geqq 2 \bar{N}_{E}\left(r, \frac{1}{f^{(n+1)}-a}\right)-S(r, f) .
$$

Combining (4.15), (4.16) with (4.11), we get

$$
\lim _{\substack{r \rightarrow \infty \\ r \notin e}} \frac{N_{E}\left(r, \frac{1}{f^{(n+1)}-a}\right)}{N\left(r, \frac{1}{f^{(n+1)}-a}\right)}=\frac{1}{2}
$$

for some finite linear measure $e$ of $r$ which contradicts the assumption of the theorem.

\section{$\S 5$. Proof of Theorem 3}

Assume that $f^{\prime}-b \not \equiv f-a$. By using the same methods as those in proof of Lemma 3 , we get that

$$
N\left(r, \frac{\mathrm{I}}{f^{\prime \prime}}\right)=S(r, f)
$$

and that

$$
T(r, f) \leqq 2 N\left(r, \frac{1}{f-a}\right)+S(r, f) .
$$


Using an argument similar to that in the proof of Theorem 2, we can assume that $f^{\prime} \not \equiv f^{\prime \prime}$. Then

$$
N\left(r, \frac{1}{f-a}\right) \leqq N\left(r, \frac{1}{\frac{f^{\prime \prime}}{f^{\prime}}-1}\right)+S(r, f) \leqq T\left(r, \frac{f^{\prime \prime}}{f^{\prime}}\right)+S(r, f) \leqq N\left(r, \frac{1}{f^{\prime}}\right)+S(r, f) .
$$

Hence

$$
T(r, f) \leqq 2 N\left(r, \frac{1}{f^{\prime}}\right)+S(r, f) .
$$

Let $z_{0} \in f^{-1}(a) \cap\left(f^{\prime}\right)^{-1}(b) \cap\left(f^{\prime \prime}\right)^{-1}(b)$. From Lemma 5 and the assumption that $f$, $f^{\prime}, f^{\prime \prime}$ share $(a, b, b)$ IMN, we get that

$$
\begin{gathered}
2 H(z)+K(z)=O\left\{\left(z-z_{0}\right)\right\}, \\
T(r, H)=S(r, f), T(r, K)=S(r, f) .
\end{gathered}
$$

Now we suppose that $2 H(z)+K(z) \not \equiv$. From (5.4) we have

$$
N\left(r, \frac{1}{f-a}\right) \leqq N\left(r, \frac{1}{2 H-K}\right)+S(r, f) .
$$

Combining with (5.2) and (5.5), we get $T(r, f) \leqq S(r, f)$ which contradicts the assumption of the theorem.

Next let $2 H(z)+K(z) \equiv 0$. Then

$$
\frac{f^{\prime \prime}(z)+f^{\prime}(z)}{f(z)-a}=\frac{2 f^{\prime \prime}(z)}{f^{\prime}(z)-b} .
$$

From (5.1) and (5.3), we choose $z_{1}$ satisying $f^{\prime}\left(z_{1}\right)=0, f^{\prime \prime}\left(z_{1}\right) \neq 0$. It follows from (5.7) that

$$
f^{\prime \prime}\left(z_{1}\right)\left(\frac{1}{f\left(z_{1}\right)-a}+\frac{2}{b}\right)=0 \text {. }
$$

Hence

$$
f\left(z_{1}\right)=a-\frac{b}{2} .
$$

By the derivative calculation on both sides of (5.7), combining with $f^{\prime}\left(z_{1}\right)=0$ and $f\left(z_{1}\right)=a-b / 2$, we get that $f^{\prime \prime}\left(z_{1}\right)=b$. Therefore

$$
\frac{1}{2} T(r, f)-S(r, f) \leqq N\left(r, \frac{1}{f^{\prime}}\right)-N\left(r, \frac{1}{f^{\prime \prime}}\right) \leqq N_{E_{1}}\left(r, \frac{1}{f^{\prime \prime}-b}\right) \leqq N_{E}\left(r, \frac{1}{f^{\prime \prime}-b}\right)
$$

where $E_{1}=E\left\{f^{\prime}, f^{\prime \prime} ;(b, b)\right\}, E=\left\{f, f^{\prime}, f^{\prime \prime} ;(a, b, b)\right\}$ which contradicts the condition that $f, f^{\prime}$ and $f^{\prime \prime}$ share the array $(a, b, b)$ IMN.

The author dedicates acknowledgements to the referee for his useful sugguestions and pertinent comments. 


\section{REFERENCES}

[1] L. Rubel ANd C.C. YANG, Values shared by entire functions and their derivatives, Complex Analysis, Kentucky 1976, Lecture Notes in Math., 599, SpringerVerlag, Berlin-Heldelberg-New York, 1977, 101-103.

[2] G. Jank, E. Mues and L. Volkman, Meromorphe funktionen, die mit ihrer ersten und $Z_{\text {weiten }}$ Ableitung einen endlichen Wert teilen, Complex Variables Theory Appl. (1), 6 (1986), 51-71.

[3] L. YANG, Value Distribution Theory and New Research on it, Science Press, Be1jing, 1982 (in Chinese).

[4] G. Frank and W. Ohlenroth, Meromorphe funktionen, die mit einer ihrer Ableitungen Werte teilen, Complex Variables Theory Appl. (1), 6 (1986), 2337.

[5] G. Gundersen, Meromorphic functions that share four values, Trans. Amer. Math. Soc., 277 (1983) 545-567. correction. 304 (1987), 847-850.

[6] G. Gundersen, Meromorphic functions that share three or four values, J. London Math. Soc. (2), 20 (1979), 457-466.

[7] G. Gundersen, Meromorphic functions that share three values IM and a fourth value CM, Complex Variables Theory Appl., 20 (1992), 99-106.

[8] W. K. Hayman, Meromorphic Functions, Clarendon Press, Oxford, 1964.

[9] H. Z HONG, On the extension of F. Nevanlinna Conjecture, Acta Math. Sinica (1), 36 (1993), 90-98.

Department of Mathematics and Physics

Nanjing University of Aeronautics and Astronautics

JiAngsu, 210016, P. R. China 